\title{
Cimentos Usados em Prótese Fixa: uma pesquisa com especialistas em prótese de Porto Alegre
}

\author{
Cements Used for Fixed Prosthodontics: a survey with Porto Alegre specialists
}

Priscila Veit Bohn ${ }^{1}$, Daniela Andrioli ${ }^{2}$, Vicente Castelo Branco Leitune ${ }^{3}$, Fabrício Mezzomo Collares ${ }^{4}$, Daniela Maffei Botega ${ }^{5}$, Daniela Meira $^{6}$, Carmem Borges Forges ${ }^{7}$, Susana Maria Werner Samuel ${ }^{8}$

\section{Abstract}

Actually, many dental cements are available to cement fixed prosthesis at dental clinical practice. The aim of this study was to evaluate the cements used to fixed prosthesis by specialists who work at city of Porto Alegre with a questionnaire. A questionnaire with 22 questions was elaborated and applied to professionals registered at Regional Dentistry Council (CRO). From the 225 electable subjects, 39 were excluded. The sample of 186 specialists, 72 weren't interviewed. The 114 interviewed subjects represented a $61.29 \%$ of response rate. The graduate year of $52 \%$ of subjects was between 1981 and 2000 , and $63 \%$ finished the post graduate course after 1991. To final cementation of fixed metal ceramic prosthesis, $65 \%$ used zinc phosphate cement. However, to cements metal free fixed prosthesis, $57 \%$ used resinous cement. With technological advances at rehabilitation materials, new materials and techniques were developed. However, the zinc phosphate cement is still the first choice to cement metal ceramic prosthesis to Porto Alegre specialists.

Keywords: Dental Prosthesis; Dental Cements; Zinc Phosphate Cement.

\section{Resumo}

Atualmente, diversos cimentos são utilizados para cimentação de próteses fixas na prática clínica odontológica. Sendo assim, o objetivo do presente estudo foi avaliar o uso de cimentos para prótese fixa por especialistas em prótese dentária que atuam no município de Porto Alegre. Para isso, um questionário com 22 perguntas foi elaborado e aplicado a tais profissionais registrados no Conselho Regional de Odontologia. Dentre os 225 indivíduos elegíveis, 39 foram excluídos. Da amostra de 186 especialistas, 72 não foram entrevistados. Os 114 indivíduos entrevistados representaram uma taxa de resposta de 61,29\%. O ano de graduação de $52 \%$ dos respondentes foi entre 1981 e 2000 e $63 \%$ concluíram o curso de pós-graduação após 1991. Para cimentação final de próteses fixas métalo-cerâmicas, 65\% dos dentistas usavam cimento de fosfato de zinco. Entretanto, para prótese fixas livres de metal, $57 \%$ utilizavam cimento resinoso. Com o avanço da tecnologia em materiais reabilitadores, novos materiais e técnicas foram criados, no entanto, o cimento de fosfato de zinco segue como primeira escolha entre os especialistas para cimentação final de próteses métalo-cerâmicas.

Palavras-chave: Prótese Dentária; Cimentos Dentários; Cimento de Fosfato de Zinco.
1 Aluna de Graduação, Laboratório de Materiais Dentários da Faculdade de Odontologia, Universidade do Rio Grande do Sul

2 Aluna de Graduação, Laboratório de Materiais Dentários da Faculdade de Odontologia, Universidade do Rio Grande do Sul

3 Aluno de Mestrado, Laboratório de Materiais Dentários da Faculdade de Odontologia, Universidade do Rio Grande do Sul

${ }^{4}$ Aluno de Doutorado, Laboratório de Materiais Dentários da Faculdade de Odontologia, Universidade do Rio Grande do Sul

5 Doutora e Professora, Departamento de Odontologia Conservadora da Faculdade de Odontologia, Universidade do Rio Grande do Su

6 Aluna de Mestrado, Laboratório de Materiais Dentários da Faculdade de Odontologia, Universidade do Rio Grande do Sul

${ }^{7}$ Doutora e Professora em Materiais Dentários, Laboratório de Materiais Dentários da Faculdade de Odontologia, Universidade do Rio Grande do Sul

8 Doutora e Professora Titular em Materiais Dentários, Laboratório de Materiais Dentários da Faculdade de Odontologia, Universidade do Rio Grande do Sul

Correspondência: Universidade do Rio Grande do Sul

Endereço: Ramiro Barcelos 2492 - CEP 90035003, Porto Alegre - RS, Brasil Fone: +55 51 3308-5198

E-mail: 1 prib.odonto@gmail.com, ${ }^{2}$ anigandrioli@hotmail.com,

${ }^{3}$ vicenteleitune@gmail.com, ${ }^{4}$ fabriciodonto@gmail.com, ${ }^{5}$ danimaffei@yahoo.com 6 danny_meira@hotmail.com, ${ }^{7}$ fortes@adufrgs.ufrgs.br,

${ }^{8}$ samuelsp@adufrgs.ufrgs.br

Data de Submissão: 04/11/2009

Data de Aceite: 20/07/2010

\section{Introdução}

O agente de cimentação de prótese fixa ideal deve apresentar biocompatibilidade, prevenir microinfiltração, ter força suficiente para resistir a forças funcionais durante a longevidade da restauração, possuir baixa solubilidade e não possuir sorção de água, ter adesividade, radiopacidade, estética, fácil manipulação e baixo custo (ROSENSTIEL; LAND; CRISPIN, 1998). Visto que nenhum agente de cimentação usado em Odontologia consegue preencher estes requisitos, a busca por um agente ideal de cimentação tem sido objetivo de diversas pesquisas (BURKE et al., 2002; ESCRIBANO; DE LA MACORRA, 2006; HEINTZE, 2010).

$O$ fosfato de zinco é o agente cimentante mais antigo em uso - introduzido em 1800- e com longa história de sucesso clínico para próteses metálicas, métalo-cerâmicas e cerâmicas (HILL, 2007). Tradicionalmente, o cimento de fosfato de zinco tem sido utilizado para a cimentação de próteses fixas devido ao fato de apresentar baixo custo em relação aos outros cimentos e ao sucesso clínico obtido (ROSENSTIEL; LAND; CRISPIN, 1998; SANTOS; KATO; CONTI, 2003). Sabe-se, no entanto, que o cimento de fosfato de zinco apresenta desvantagens já bem documentadas (POWERS; DENNISON, 1974; GRIEVE, 1969) como a sua acidez e alta solubilidade, que fazem aumentar a possibilidade de ocorrer infiltração marginal (PEGORARO et al., 1998).

Com o advento de novas opções para a cimentação de peças protéticas, o cimento de fosfato de zinco deixou de ser a única escolha. Atualmente, algumas restaurações protéticas, como 
inlays e onlays, exigem uma cimentação adesiva para uma maior retenção da restauração (HILL, 2007; HEINTZE, 2010). Contudo próteses métalo-cerâmicas, por exemplo, dificultam a cimentação adesiva, tendo em vista que a estrutura metálica não permite a passagem de luz necessária para a ativação do cimento resinoso. Ainda assim, existem possibilidades de cimentação com outros materiais, como os cimentos de ionômero de vidro e ionômero de vidro modificado por resina (HEINTZE, 2010). Recentemente, cimentos auto-adesivos tem ganhado popularidade pela sua fácil aplicação e por suas propriedades adesivas. Entretando, sua adesão é menor que outros materiais que utilizam sistema adesivo (ESCRIBANO; DE LA MACORRA, 2006; YANG et al., 2006).

Essas opções mais recentes como o cimento resinoso e o cimento de ionômero de vidro modificado por resina apresentam algumas vantagens como a baixa solubilidade e a adesão à estrutura dental. Esta adesão reforça a estrutura dental remanescente conferindo maior longevidade à restauração protética além de permitir cimentações estéticas. Contudo estes cimentos apresentam uma maior viscosidade o que pode dificultar o assentamento da peça protética, causando desadaptações marginais e interferências oclusais (ROSENSTIEL; LAND; CRISPIN, 1998). Além disso, possuem uma maior sensibilidade técnica, dificultando o procedimento clínico.

Com o avanço da tecnologia na Odontologia e o surgimento desses novos agentes de cimentação de prótese fixa, a frequência com que estes materiais passaram a substituir os já existentes é desconhecida. Portanto, para que se conheça o impacto da inovação tecnológica na clínica odontológica, o objetivo do presente estudo foi avaliar o uso de cimentos para prótese fixa por especialistas em prótese dentária que atuam no município de Porto Alegre.

\section{Métodos}

Fizeram parte desde estudo cirurgiões dentistas especialistas em prótese dentária, registrados no Conselho Regional de Odontologia (CRO/RS), que atuassem no município de Porto Alegre. O trabalho foi aprovado pelo Comitê de Ética e Pesquisa da Faculdade de Odontologia da Universidade Federal do Rio Grande do Sul (256/07). A relação dos cirurgiões dentistas e respectivos endereços foram fornecidos pelo CRO/RS. Os participantes não foram identificados, nem o teor das respostas ao questionário, garantindo total sigilo dos mesmos. Os critérios de exclusão foram: aposentadoria, mudança de endereço e impossibilidade de localização. Os questionários foram entregues individualmente em cada consultório juntamente com 0 Consentimento Informado, o qual os cirurgiões dentistas foram instruídos a ler e assinar, se estivessem de acordo, antes de participarem da pesquisa.

O questionário foi construído baseado em questionários adaptados da literatura (SANTOS; KATO; CONTI, 2003) contendo 22 questões, sendo 12 delas abertas e 10 fechadas (Quadro 1) Apresenta dois blocos de perguntas: Dados Pessoais com 8 questões e Informações Profissionais com mais 14 questões. O questionário foi previamente testado com especialistas que não atuavam no município de Porto Alegre quanto a sua compreensão, interpretação e plausibilidade de aplicação na população pretendida. Decorrida uma semana da data da entrega dos questionários, os especialistas eram contatados por telefone para a confirmação de que haviam respondido o questionário, permitindo a devolução. Os profissionais que não responderam a três tentativas semanais consecutivas, de contato, foram excluídos da pesquisa.

\section{Resultados e Discussão}

O total de cirurgiões dentistas especialistas em prótese dentária registrados no $\mathrm{CRO} / \mathrm{RS}$ foi de 225 , dos quais 39 foram excluídos segundo os critérios previamente descritos: 9 eram aposentados, 6 haviam se mudado e 18 não foram encontrados. Dessa amostra de 186 especialistas, 11 recusaram-se a responder e 61 não responderam à terceira ligação, totalizando 114 especialistas entrevistados, resultando em uma taxa de resposta de $61,29 \%$. Estudo semelhante reportado na literatura (SHINKKAI et al., 2000) mostra uma taxa de 19\%, evidenciando a alta taxa de resposta obtida no presente estudo.

As figuras de 1 e 2 apresentam dados que caracterizam a população dos especialistas em prótese, quanto à idade, gênero, renda e anos de graduação e pós-graduação. A metade dos entrevistados possuía de 31 a 50 anos (Figura 1A), a maioria (68\%) era do gênero masculino (Figura $1 \mathrm{~B}$ ), de renda maior do que $\mathrm{R} \$$ $4000,00(77 \%)$ (Figura 1C), se formou entre 1981 e 2000 (Figura 2A) e finalizou sua especialização a partir de 1991 (Figura 2B). Quanto ao local de graduação observou-se que $48 \%$ concluíram a graduação na UFRGS, $41 \%$ em outras universidades da região metropolitana de Porto Alegre, $5 \%$ no interior do Estado, $2 \%$ fora do Estado e 4\% não responderam. No que se refere à pósgraduação observou-se uma maior distribuição entre diversas universidades, $\mathrm{ABO}$ e CFO, sendo que, $89 \%$ graduaram-se em universidades da Região Metropolitana de Porto Alegre.

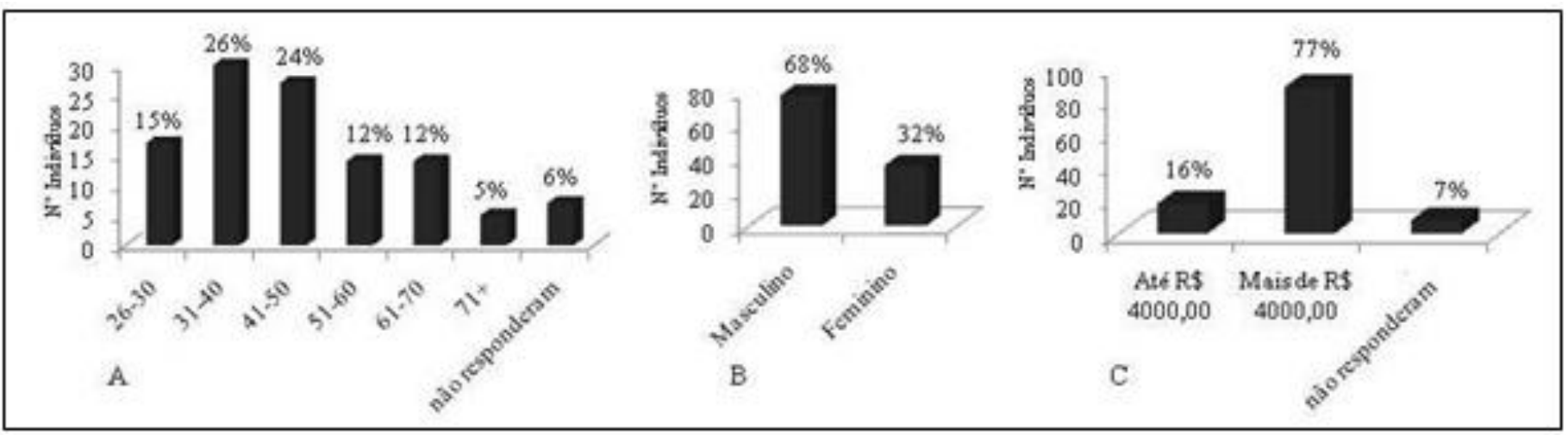

Figura 1. A: Idade; B: Gênero; C: Renda dos protesistas. 


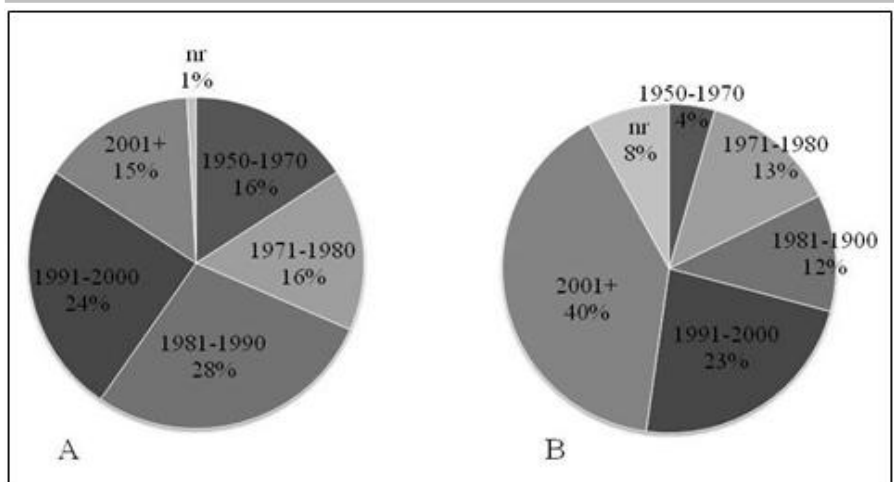

Figura 2. A: Ano da Graduação; B: Ano da pós-graduação dos especialistas.

Quanto ao tipo de prótese fixa mais utilizada pelos entrevistados, $80 \%$ responderam utilizar prótese fixa métalocerâmica e $8 \%$, prótese fixa livre de metal (Figura 3A). Para aqueles que utilizam métalo-cerâmica com maior freqüência, o cimento de fosfato de zinco é o cimento de escolha em $65 \%$ das vezes (Figura 3B). O fosfato de zinco não possui adesividade $e$ consegue alcançar suas propriedades físicas máximas em 24 horas. Sua

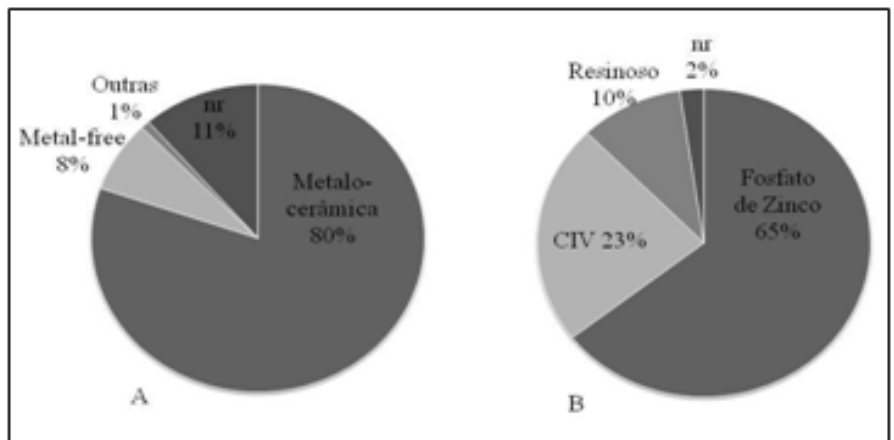

Figura 3. A: Tipo de prótese fixa que os especialistas mais utilizam; $\mathrm{B}$ : Cimento que os profissionais mais utilizam para cimentar próteses metalo-cerâmicas. nr: não responderam. resistência a compressão é muito alta, porém a resistência a tração é baixa comparada com outros cimentos disponíveis. Possui um alto módulo de elasticidade e sua solubilidade inicial é alta, mas diminui rapidamente. Fosfato de zinco é o agente de cimentação de menor custo no mercado (HILL, 2007).

Já para aqueles que utilizam próteses livres de metal com maior frequência, o cimento resinoso é o cimento de escolha na maioria das vezes (57\%). Esses dados podem ser explicados com o seguinte resultado: a maior parte dos especialistas tinha o tipo de prótese $(52 \%)$ como critério de escolha do cimento para cimentar prótese fixa (Figura 4A). Cimentos resinosos se aderem quimicamente a porcelanas condicionadas e silanizadas (DIAZARNOLD; VARGAS; HASELTON, 1999). Em geral, esses cimentos são considerados a melhor escolha para cimentar restaurações livres de metal, baseado em vários estudos laboratoriais e clínicos de acordo com resistência a fratura e selamento (BURKE et al., 2002). Resistência a tração, resistência a fratura e resiliência de cimentos resinosos são iguais ou maiores que outros agentes de cimentação; a solubilidade é baixa e as características estéticas são boas, com possibilidade de escolha de cor. No entanto, os agentes de cimentação resinosos não liberam flúor, sua espessura de filme é relativamente alta, seu módulo de elasticidade é alto, possui elevada sensibilidade técnica e um custo mais alto que os cimentos convencionais (ROSENSTIEL; LAND; CRISPIN, 1998; ZHEN; WHITE, 1999; MOUNT, 2002)

O cimento de fosfato de zinco foi também o mais escolhido pelos especialistas para cimentar uma prótese fixa que seria usada em sua própria boca, caso precisassem utilizar prótese fixa, como se pode observar na Figura 4B. Os achados do presente estudo concordam com a literatura na elevada taxa de escolha das próteses fixas métalo-cerâmica e a alta freqüência de uso do fosfato de zinco (SHINKKAI et al., 2000; CHRISTENSEN, 1997). E, segundo Heintze, em 2010, os cimentos resinosos levam a um maior número de falhas por tensão do que cimentos de ionômero de vidro, que por sua vez apresentam, na maioria das vezes, um maior número de falhas que cimentos de fosfato de zinco.

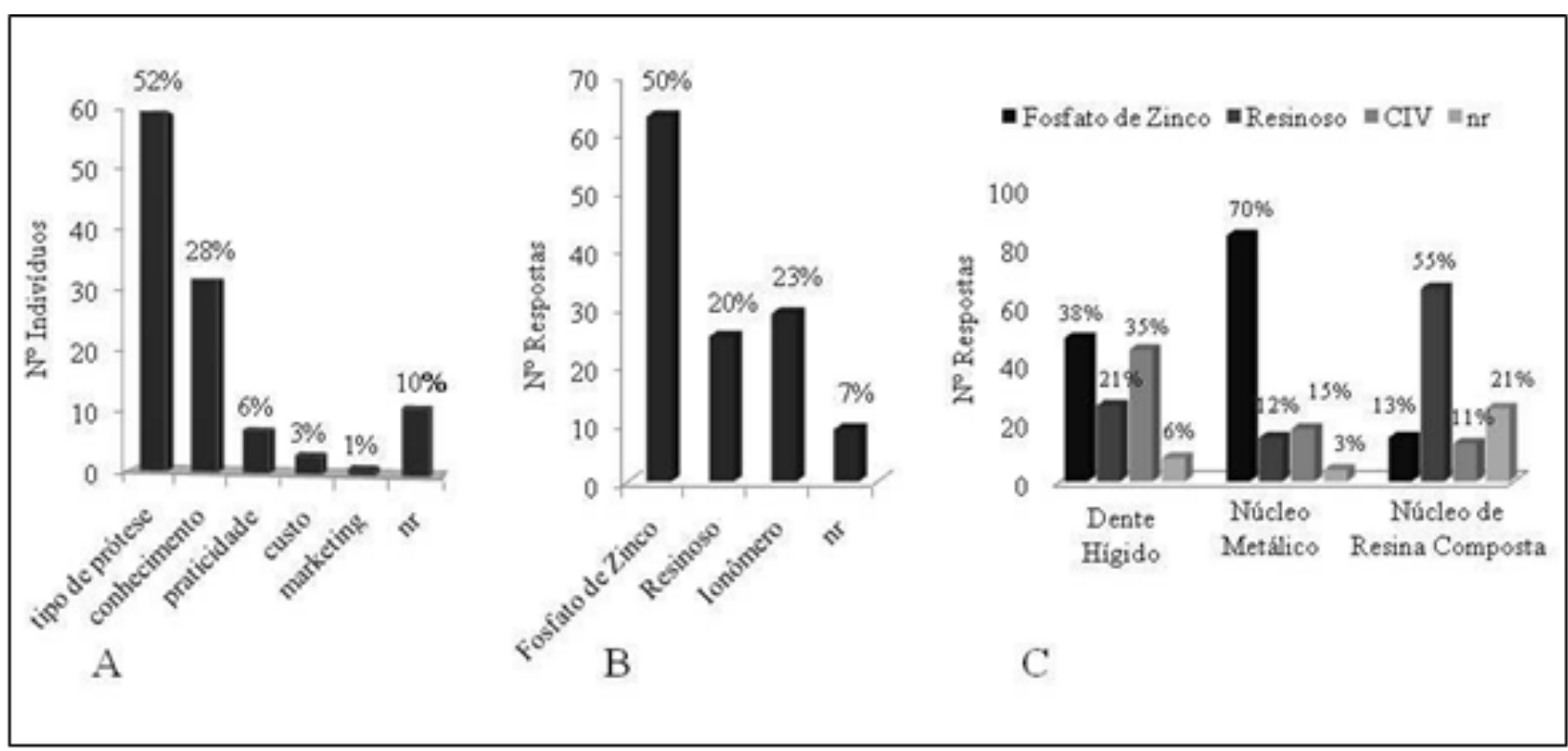

Figura 4. A: Critério de escolha do cimento para cimentar prótese fixa; B: Cimento escolhido pelos especialistas para cimentar uma prótese fixa que seria usada em sua própria boca, se precisassem utilizar prótese fixa. nr: não responderam. 
No entanto, para os dados de qual cimento utilizar em sua própria boca, os resultados do presente estudo concordam com o os dados de Shinkkai et al. (2000), e discordam dos dados de Christensen (1997). Uma possível explicação seria a diferença demográfica entre as populações estudadas: enquanto os entrevistados de Shinkai et al eram brasileiros, os entrevistados de Christensen eram norte-americanos. Uma provável diferença de filosofias de ensino poderia explicar estes resultados conflitantes. Os especialistas em prótese dentária brasileiros se mostram mais conservadores na escolha do material a ser adotado de acordo com o presente estudo e o de Shinkkai et al., em 2000.

Outro fator estudado foi a influência do substrato sobre o qual a prótese iria ser cimentada. A Figura $4 \mathrm{C}$ apresenta os cimentos preferidos para cimentar próteses sobre diferentes substratos, como dente hígido, núcleo metálico e núcleo de resina composta. Para cimentar próteses sobre dente hígido e sobre núcleo metálico, o fosfato de zinco foi o cimento de escolha em 38 e $70 \%$ das vezes, respectivamente. Enquanto que para cimentar prótese sobre o núcleo de resina composta, o cimento resinoso foi o cimento escolhido (55\%). Uma explicação para este fato pode ser o amplo conhecimento do comportamento clínico do cimento de fosfato de zinco, pequena sensibilidade técnica (DONOVAN et al., 2004 DONOVAN; CHO, 1999; DONOVAN, 2006; HEINTZE, 2010) e a experiência adquirida com o uso do cimento pelo profissional visto que todos entrevistados aprenderam a usar cimento de fosfato de zinco na graduação (Figura 5A).

$\mathrm{Na}$ Figura 5B é demonstrado um aumento na freqüência do uso dos cimentos de ionômero de vidro e resinosos no curso de pós-graduação, provavelmente, devido ao fato destes cimentos terem sido lançados mais recentemente no mercado. No entanto, em sua prática diária os entrevistados preferem o uso de fosfato de zinco, quando não há contra-indicações já consagradas como próteses adesivas e algumas próteses que são livres de metal.

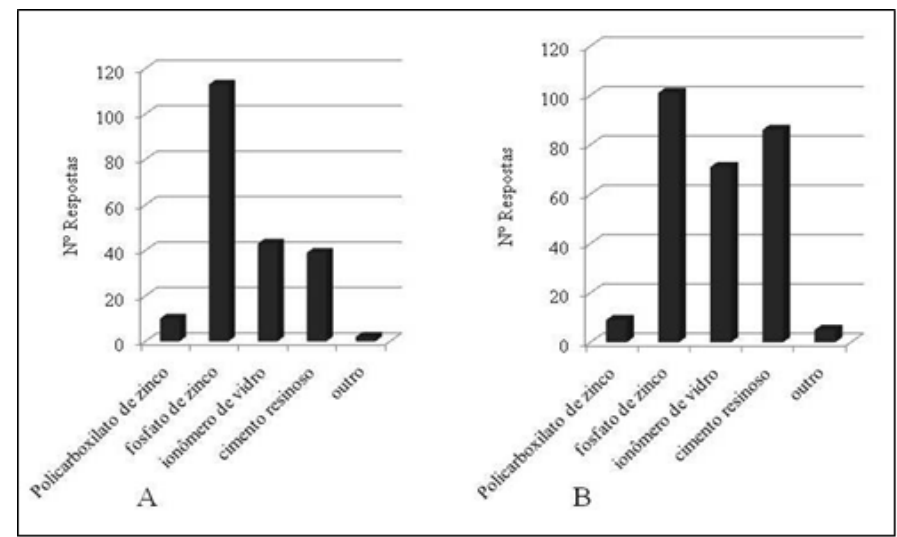

Figura 5. A: Cimentos que os especialistas aprenderam a utilizar na graduação; B: Cimentos que aprenderam a utilizar na pósgraduação.

Daqueles que aprenderam a utilizar fosfato de zinco no curso de pós-graduação, $80 \%$ ainda utilizava e 19\% não utilizava mais fosfato de zinco para cimentação de prótese fixa. Esses dados foram cruzados com a freqüência com que os especialistas participavam de congressos, seminários ou cursos, observando-se que aqueles que não utilizavam mais fosfato de zinco têm uma freqüência maior nesses eventos - $84 \%$ afirmaram ir mais de uma vez ao ano nesses eventos, enquanto $65 \%$ daqueles que ainda usavam fosfato de zinco alegaram ir menos de uma vez ao ano nesses eventos. Uma explicação para esse fato poderia ser que nos congressos e cursos os cirurgiões dentistas têm contato com novas evidências de novos materiais diferentes que o cimento de fosfato de zinco ou ainda os cirurgiões dentistas podem ser influenciados pelo marketing de outros materiais.

\section{Conclusão}

Embora haja uma grande oferta de novos cimentos e a inovação tecnológica esteja bastante presente nos materiais dentários, o cimento de fosfato de zinco mostrou-se o cimento mais utilizado para cimentar próteses fixas por especialistas que atuam no município de Porto Alegre.

\section{Referências}

BURKE, F. J. et al. Are adhesive technologies needed to support ceramics? An assessment of the current evidence. J. Adhes. Dent., New Malden, Surrey, v. 4, no. 1, p. 7- 22, 2002.

CHRISTENSEN, G. J. Cements used for full crown restorations: a survey of the American Academy of Esthetic Dentistry. J. Esthet. Dent., Philadelphia, v. 9, no. 1, p. 20-26, Jan. 1997.

DIAZ-ARNOLD, A. M.; VARGAS, M. A.; HASELTON, D. R. Current status of luting agents for fixed prosthodontics. J. Prosthet. Dent., St. Louis, v. 81, no. 2, p. 135-141, Feb. 1999.

DONOVAN, T. E. The selection of contemporary restorative materials: anecdote vs. evidence-based? J. Calif. Dent. Assoc. Sacramento, CA, v. 34, no. 2, p. 129-134, Feb. 2006.

DONOVAN, T. E.; CHO, G. C. A contemporary evaluation of dental cements. Compend. Contin. Educ. Dent., Jamesburg, N.J., v. 20, no. 3, p. 197-199, Mar. 1999.

DONOVAN, T. et al. Retrospective clinical evaluation of 1,314 cast gold restorations in service from 1 to 52 years. J. Esthet. Restor. Dent., Hamilton, Ontario, v. 16, no. 3, p. 194-204, May 2004.

ESCRIBANO, N.; DE LA MACORRA, J.C. Microtensile bond strength of self-adhesive luting cements to ceramic. J. Adhes. Dent., New Malden, Surrey, v. 8, no. 5, p. 337-341, Oct. 2006.

GRIEVE, A. R. A study of dental cements. Br. Dental J., London, v. 127 , p. $405-410,1969$.

HEINTZE, S. D. Crown pull-off test (crown retention test) to evaluate the bonding effectiveness of luting agents. Dent. Mater. Copenhagen, v. 26, no. 3, p. 193-206, Mar. 2010.

HILL, E. E. Dental cements for definitive luting: a review and practical clinical considerations. Dent. Clin. North Am., Philadelphia, v. 51, no. 3, p. 643-658, July 2007.

MOUNT, G. J. An atlas of glass-ionomer cements, a clinicians guide. 3rd ed. New York: Martin Duntiz, 2002.

PEGORARO, L.F. et al. Prótese fixa. São Paulo: Artes Médicas, 1998.

POWERS, J. M.; DENNISON, J. D. A review of dental cements used for permanent retention of restorations Part II: propertie and criteria for selection. J. Mich. Dental Assoc., Lansing, MI, v. 56, p. 218-225, 1974

ROSENSTIEL, S.F.; LAND, M.F.; CRISPIN, B.J. Dental luting agents: a review of the current literature. J. Prosthet. Dent., St. Louis, v. 80, no. 3, p. 280-301, Sept. 1998.

SANTOS, C. N.; KATO, M. T.; CONTI, P. C. R. Avaliação das condutas adotadas por profissionais na utilização de coroas metalocerâmicas. J. Appl. Oral Sci., Bauru, v. 11, no. 4, p. 290-300, Oct./Dec. 2003.

SHINKKAI, R. S. A. et al. Materiais dentários utilizados em prótese: pesquisa entre protesistas brasileiros. R. Gaúcha Odontol., Porto Alegre, v. 48, n. 2, p. $77-81,2000$ 
YANG, B. et al. Micro-tensile bond strength of three luting resins to human regional dentin. Dent. Mater., Copenhagen, v. 22, no. 1, p. 45-56, Jan. 2006.
ZHEN, C. L.; WHITE, S. Mechanical properties of dental luting cements. J. Prosthet. Dent., St. Louis, v. 81, no. 5, p. 597-609, May 1999.

Quadro 1: Questionário aplicado no estudo.

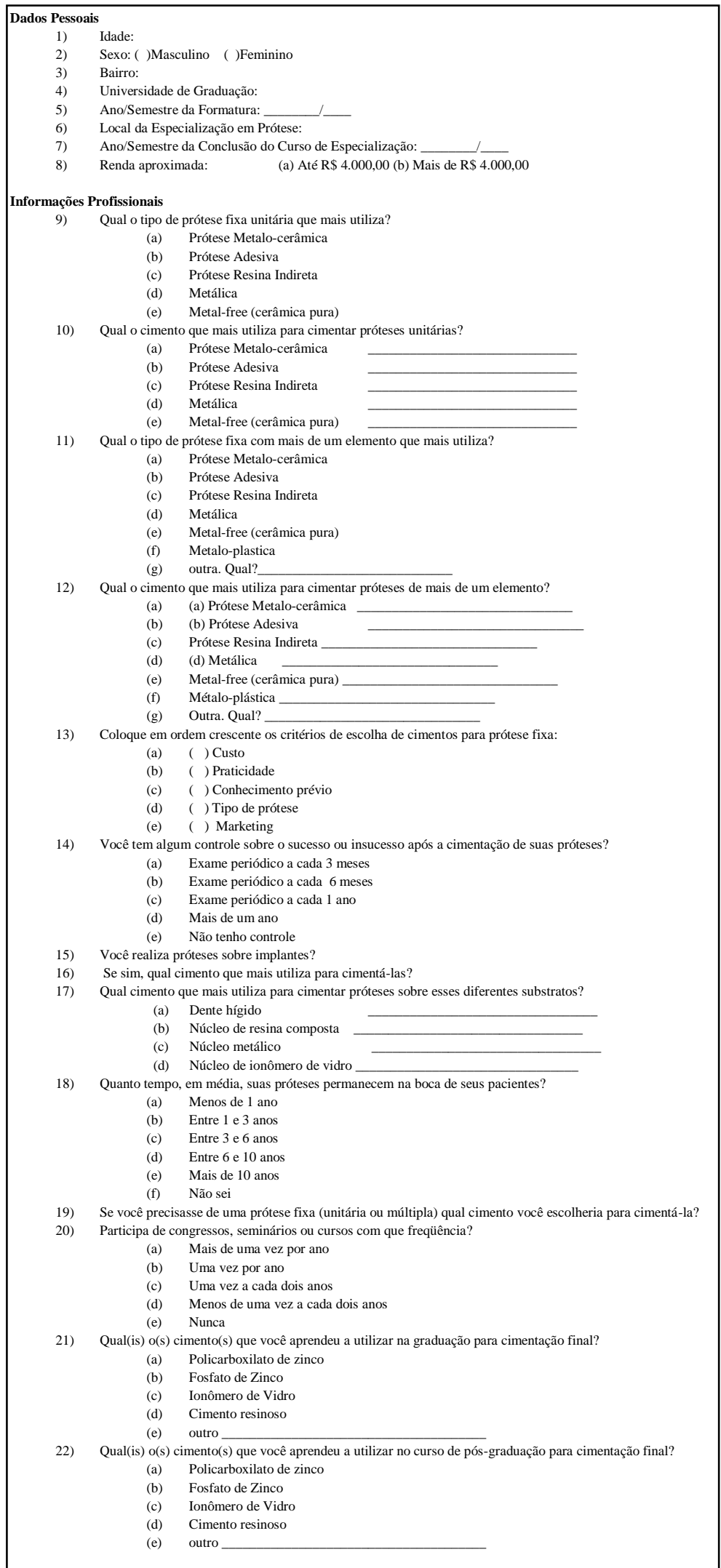

